\title{
TRANSFER OF ESTIMATES FROM CONVEX TO STRONGLY PSEUDOCONVEX DOMAINS IN $\mathbb{C}^{N}$
}

\author{
ADIB A. FADLALLA \\ Department of Mathematics \\ Faculty of Science, Cairo University \\ Giza, Egypt
}

\begin{abstract}
In this article, estimates of the hyperbolic and Carathéodory distances in domains $G \subset \subset \mathbb{C}^{n}, n \geq 1$, are obtained. They are equally valid for the Kobayashi distance.
\end{abstract}

1. Introduction. In Section 2, general definitions and notions are given, upper and lower estimates of the hyperbolic distance $C_{E_{r}}(z, w), z, w \in E_{r}$ in the disc $E_{r} \subset \mathbb{C}$ with radius $r$ are obtained, which show that these estimates depend mainly on mutual ratios of the distances $d\left(z, \partial E_{r}\right), d\left(w, \partial E_{r}\right)$ of $z, w$ from the boundary $\partial E_{r}$ of $E_{r}$ and their distance apart $|z-w|$.

These results are used in Section 3 to obtain estimates in the Euclidean ball $B_{r} \subset \mathbb{C}^{n}$, $n>1$, using the idea that: if $z, w \in B_{r}$ then the plane section $D$ of $B_{r}$ by the 1dimensional analytic complex plane through $z, w$ is a metric plane in the terminology of Carathéodory [2] or a geodesic in the terminology of Vesentini [7]. Sufficient and necessary conditions for the boundedness of $C_{B_{r}}(z, w)$ are obtained. An example shows that the necessary and sufficient condition for $E_{r} \subset \mathbb{C}$ is only sufficient but not necessary in $B_{r} \subset \mathbb{C}^{n}, n>1$.

In Section 4, it is shown that it is possible to use chains of balls to get estimates of $C_{G}(z, w)$ in a domain $G \subset \subset \mathbb{C}^{n}$, under certain conditions; which are easily applied if $G$ is convex. The estimates is then transfered from convex domains to strongly pseudoconvex domains by means of local biholomorphic maps [1, page 132]. A sufficient condition (but not necessary) for the boundedness of $C_{G}(z, w), z, w \in G$ is obtained. An example is given.

Finally, in section 5, the continuous extension of biholomorphic maps between strongly pseudoconvex domains with $C^{2}$ boundaries is proved [cf. 8].

1991 Mathematics Subject Classification: Primary 32F15; Secondary 32H15.

The paper is in final form and no version of it will be published elsewhere. 


\section{Basic notions}

DEFinitions:

1- Let $G \subset \subset \mathbb{C}^{n}$ be a domain $G$ is called smooth if for every $z_{o} \in \partial G$ (the boundary of $G$ ) there exist a neighbourhood $U$ of $z_{o}$ and a real valued function $f \in C^{2}(U)$ such that

$$
G \cap U=\{z \in U: f(z)<0\} \text { and } \quad d f \neq 0 \text { in } G \cap U .
$$

2- In definition (1), the 1-dimensional real inward normal to $\partial G$ at $z_{o}$, will be denoted by $N_{z_{0}}$.

3- If $z=\left(z_{1}, \cdots, z_{n}\right) \in \mathbb{C}^{n}$, then $|z|=\sqrt{\left|z_{1}\right|^{2}+\cdots+\left|z_{n}\right|^{2}}$.

4- If in definition (1), $z^{\prime} \in G$, then $d\left(z^{\prime}, \partial G\right)$ denotes the distance of $z^{\prime}$ from $\partial G$. Obvious there is a point $z_{o}^{\prime} \in \partial G$ such that $z^{\prime} \in N_{z_{o}^{\prime}}, z_{o}^{\prime}$ will be called the projection of $z^{\prime}$ on $\partial G$.

5- Let $G \subset \mathbb{C}^{n}$ be a domain, $U \subset \mathbb{C}^{n}$, then $\partial G \cap U$ will be denoted by $\beta_{U}$.

6 - If $z, w \in \mathbb{C}^{n}$, then the 1-dimensional complex analytic plane through $z$, $w$ will be denoted by $P(z, w)$.

7- Let $B_{r} \subset \mathbb{C}^{n}$ be the ball $|z|<r, z \in \mathbb{C}^{n}$. Let $G \subset \subset \mathbb{C}^{n}$ be a smooth domain and $z_{o} \in \partial G$, then $B_{r}$ when placed tangential to $\partial G$ at $z_{o}$, with $N_{z_{o}}$ lying on a diameter will be denoted by $B_{r}^{\left(z_{o}\right)}$. If $B_{r}^{\left(z_{o}\right)} \subset G$ for all $z_{o} \in \partial G$, then $B_{r}$ is called an admissible ball to $G$. If $G \subset \subset \mathbb{C}^{n}$ is smooth, then there exist always admissible balls $B_{r}$.

If $z_{o}$ has a neighbourhood $U$ such that $B_{r}^{(w)} \subset G$ for all $w \in \beta_{U}$, then $B_{r}$ is called an admissible ball to $G$ at $z_{o}$.

8- Let $G \subset \subset \mathbb{C}^{n}, z, w \in G$; the Carathéodory and Kobayashi Distances in $G$ will be denoted by $C_{G}(z, w)$ and $K_{G}(z, w)$ respectively.

Proposition 2.1. Let $D, D_{1} \subset \mathbb{C}^{n}$ be domains, $D^{\prime} \subset \subset D, D_{1}^{\prime} \subset \subset D_{1}$ and $\phi$ a biholomorphic map of $D$ onto $D_{1}, \phi\left(D^{\prime}\right)=D_{1}^{\prime}, z^{(j)} \in D^{\prime}, j=1,2$ and $\phi\left(z^{(j)}\right)=w^{(j)} \in$ $D_{1}^{\prime}, j=1,2, \ell_{1}=\left|z^{(1)}-z^{(2)}\right|, \ell_{2}=\left|w^{(1)}-w^{(2)}\right|$. If the line segments

$$
L_{1}=\overrightarrow{z^{(1)} z^{(2)}}, L_{2}=\overrightarrow{w^{(1)} w^{(2)}}, L_{1} \subset D^{\prime}, L_{2} \subset D_{1}^{\prime}
$$

then, there exist $0<\alpha<\beta<+\infty$ such that $\alpha<\left(\ell_{1} / \ell_{2}\right)<\beta$.

Proof. Let $v=z^{(2)}-z^{(1)}=\left(v_{1}, \cdots, v_{n}\right), \gamma=\phi\left(L_{1}\right), \phi=\left(\phi_{1}, \cdots, \phi_{n}\right), \ell_{2}^{\prime}=$ length of the curve $\gamma,\left(d \phi_{j}, v\right)=\sum_{\mu=1}^{n} \frac{\partial \phi_{j}}{\partial z_{\mu}} v_{\mu}$. Since $\phi$ is regular in $D$ and $v \neq 0,\left|d \phi_{j}\right|_{D^{\prime}} \neq$ $0, j=1, \cdots, n$ and $\left(d \phi_{j}, v\right), j=1, \cdots, n$, do not vanish simultaneously. Let $M=$ $\max _{D^{\prime}}\left\{\left|d \phi_{j}\right|, j=1,2, \cdots, n\right\}$. Now, $\left|\left(d \phi_{j}, v\right)\right| \leq\left|d \phi_{j}\right| \cdot|v| \leq M \ell_{1}$, thus

$$
\ell_{2} \leq \ell_{2}^{\prime}=\int_{0}^{1} \sqrt{\sum_{j=1}^{n}\left|\left(d \phi_{j}, v\right)\right|^{2}} d t \leq \sqrt{n} M \ell_{1}=k_{1} \ell_{1}
$$

Similarly, there exist $k_{2}>0$ such that $\ell_{1} \leq k_{2} \ell_{2}$, thus, $1 / k_{1} \leq \ell_{1} / \ell_{2} \leq k_{2}$.

Proposition 2.2. In Proposition 2.1, let $H \subset D^{\prime}, H_{1} \subset D_{1}^{\prime}$ be smooth hypersurfaces such that $H_{1}=\phi(H)$. Furthermore, let $z \in D^{\prime}, w=\phi(z), n_{1}=d(z, H), n_{2}=d\left(w, H_{1}\right)$, 
then $1 / k_{1} \leq\left(n_{1} / n_{2}\right) \leq k_{2}$ provided that the projection of $z$ on $H$ is $\in H$ and projection of $w$ on $H_{1}$ is $\in H_{1}$.

Proof. Let $z_{o} \in H$ and $w_{o} \in H_{1}$ be such that $n_{1}=\left|z_{o}-z\right|, n_{2}=\left|w_{o}-w\right|$. Let $\phi\left(z_{o}\right)=w_{o}^{\prime} \in H_{1}, \phi^{-1}\left(w_{o}\right)=z_{o}^{\prime} \in H, \ell_{1}=\left|z-z_{o}^{\prime}\right|, \ell_{2}=\left|w-w_{o}\right|$. From Proposition 2.1, we have

$$
\frac{1}{k_{1}} \leq \frac{n_{1}}{\ell_{2}} \leq \frac{n_{1}}{n_{2}} \text { and } k_{2} \geq \frac{\ell_{1}}{n_{2}} \geq \frac{n_{1}}{n_{2}}
$$

Proposition 2.3. Let $E_{r} \subset \mathbb{C}$ be the disc $|z|<r$, and $z, w \in E_{r}$, then

$$
C_{E_{r}}(z, w)=\log \left[\sqrt{1+\frac{r^{2}}{\left(2 r-r_{1}\right)\left(2 r-r_{2}\right)} \cdot \frac{R^{2}}{r_{1} r_{2}}}+\frac{r}{\sqrt{\left(2 r-r_{1}\right)\left(2 r-r_{2}\right)}} \frac{R}{\sqrt{r_{1} r_{2}}}\right] \text {, }
$$

where $R=|z-w|, r_{1}=d\left(z, \partial E_{r}\right), r_{2}=d\left(w, \partial E_{r}\right)$.

Corollary 2.1. Obviously,

$$
\frac{1}{4}<\frac{r^{2}}{\left(2 r-r_{1}\right)\left(2 r-r_{2}\right)} \leq 1
$$

Thus

$$
\log \left[\sqrt{1+\frac{1}{4} \frac{R^{2}}{r_{1} r_{2}}}+\frac{1}{2} \frac{R}{\sqrt{r_{1} r_{2}}}\right]<C_{E_{r}}(z, w) \leq \log \left[\sqrt{1+\frac{R^{2}}{r_{1} r_{2}}}+\frac{R}{\sqrt{r_{1} r_{2}}}\right],
$$

which are inequalities independent on $r$, they depend only on ratios $\left(R / r_{j}\right), j=1,2$

Corollary 2.2. Let $\left\{z_{\nu}\right\}_{\nu=1}^{\infty},\left\{w_{\nu}\right\}_{\nu=1}^{\infty} \subset E_{r}$. The necessary and sufficient conditions for $\left\{C_{E_{r}}\left(z_{\nu}, w_{\nu}\right)\right\}$ to be bounded, is that there exists

$$
0 \leq M<+\infty \text { such that } \frac{R_{\nu}^{2}}{r_{\nu} r_{\nu}^{\prime}} \leq M \text { for all } \nu
$$

where $R_{\nu}=\left|z_{\nu}-w_{\nu}\right|, r_{\nu}=d\left(z_{\nu}, \partial E_{r}\right), r_{\nu}^{\prime}=d\left(w_{\nu}, \partial E_{r}\right)$.

The condition $\left(R_{\nu}^{2} / r_{\nu} r_{\nu}^{\prime}\right) \leq M$ is equivalent to $\left(R_{\nu} / r_{\nu}\right) \leq M_{1}<+\infty$ and $\left(R_{\nu} / r_{\nu}^{\prime}\right) \leq$ $M_{2}<+\infty$, for all $\nu$.

\section{The Euclidean ball}

Theorem 3.1. Let $B_{r} \subset \mathbb{C}^{n}, n>1$, be the ball $|z|<r, z \in \mathbb{C}^{n}$. If $z, w \in B_{r}$, then $C_{B_{r}}(z, w)=C_{D}(z, w)$, where $D$ is the disc $P(z, w) \cap B_{r}$ (see definition 6$)$.

Proof. Let $z=\left(z_{1}, \cdots, z_{n}\right) \in \mathbb{C}^{n}$. Obvious if $z^{\prime}=\left(z_{1}^{\prime}, 0, \cdots, 0\right), z^{\prime \prime}=\left(z_{1}^{\prime \prime}, 0, \cdots, 0\right)$, $\left|z_{1}^{\prime}\right|<r,\left|z_{1}^{\prime \prime}\right|<r$ and $D^{\prime}$ be the disc $\left|z_{1}\right|<r, z_{j}=0, j=2, \cdots, n$. Then

$$
C_{B_{r}}\left(z^{\prime}, z^{\prime \prime}\right)=C_{D^{\prime}}\left(z_{1}^{\prime}, z_{1}^{\prime \prime}\right)
$$

Now, there exists [3] an automorphism of $B_{r}$, which maps $D$ conformally on $D^{\prime}$, which proves the theorem.

From Proposition 2.3, Corollaries 2.1 and 2.2 we get: 
Corollary 3.1.

$C_{B_{r}}(z, w)=\log \left(\sqrt{1+\frac{\rho^{2}}{\left(2 \rho-\rho_{1}\right)\left(2 \rho-\rho_{2}\right)} \cdot \frac{R^{2}}{\rho_{1} \rho_{2}}}+\frac{\rho}{\sqrt{\left(2 \rho-\rho_{1}\right)\left(2 \rho-\rho_{2}\right)}} \cdot \frac{R}{\sqrt{\rho_{1} \rho_{2}}}\right)$

where $\rho=$ radius of $D, \rho_{1}=d(z, \partial D), \rho_{2}=d(w, \partial D)$ and $\frac{1}{4}<\frac{\rho^{2}}{\left(2 \rho-\rho_{1}\right)\left(2 \rho-\rho_{2}\right)} \leq 1$. Thus

$$
\log \left(\sqrt{1+\frac{1}{4} \frac{R^{2}}{\rho_{1} \rho_{2}}}+\frac{1}{2} \frac{R}{\sqrt{\rho_{1} \rho_{2}}}\right)<C_{B_{r}}(z, w) \leq \log \left(\sqrt{1+\frac{R^{2}}{\rho_{1} \rho_{2}}}+\frac{R}{\sqrt{\rho_{1} \rho_{2}}}\right)
$$

Corollary 3.2. Let $\left\{z_{\nu}\right\}_{\nu=1}^{\infty},\left\{w_{\nu}\right\}_{\nu=1}^{\infty} \subset B_{r}$; the necessary and sufficient condition for $\left\{C_{B_{r}}\left(z_{\nu}, w_{\nu}\right)\right\}$ to be bounded is that there exists

$$
0 \leq M<+\infty \text { such that } \frac{R_{\nu}^{2}}{\rho_{\nu} \rho_{\nu}^{\prime}} \leq M
$$

where $R_{\nu}=\left|z_{\nu}-w_{\nu}\right|, \rho_{\nu}=d\left(z_{\nu}, \partial D_{\nu}\right), \rho_{\nu}^{\prime}=d\left(w_{\nu}, \partial D_{\nu}\right)$, or equivalently $\left(R_{\nu} / \rho_{\nu}\right) \leq$ $M^{\prime}<+\infty, \rho_{\nu} \leq \rho_{\nu}^{\prime}$, where $D_{\nu}=P\left(z_{\nu}, w_{\nu}\right) \cap B_{r}$.

(Notice that $\rho_{\nu}+R_{\nu} \geq \rho_{\nu}^{\prime}, \rho_{\nu}^{\prime}+R_{\nu} \geq \rho_{\nu}$ ).

Corollary 3.3. From Corollary 3.1, it follows that

$$
C_{B_{r}}(z, \nu)=\log \left(\sqrt{1+\frac{\rho}{\left(2 r-r_{1}\right)\left(2 r-r_{2}\right)} \cdot \frac{R^{2}}{r_{1} r_{2}}}+\frac{\rho}{\sqrt{\left(2 r-r_{1}\right)\left(2 r-r_{2}\right)}} \cdot \frac{R}{\sqrt{r_{1} r_{2}}}\right)
$$

where $r_{1}=d\left(z, B_{r}\right), r_{2}=d\left(w, \partial B_{r}\right)$. It is obvious that

Thus,

$$
0<\frac{\rho^{2}}{\left(2 r-r_{1}\right)\left(2 r-r_{2}\right)} \leq 1 \text {. }
$$

$$
C_{B_{r}}(z, w) \leq \log \left(\sqrt{1+\frac{R^{2}}{r_{1} r_{2}}}+\frac{R}{\sqrt{r_{1} r_{2}}}\right) .
$$

Corollary 3.4. In Corollary 3.2, let $r_{\nu}=d\left(z_{\nu}, \partial B_{r}\right), r_{\nu}^{\prime}=d\left(w_{\nu}, \partial B_{r}\right)$.

From Corollary 3.3 we get:

For $\left\{C_{B_{r}}\left(z_{\nu}, w_{\nu}\right)\right\}$ to be bounded it is sufficient that there exists $0 \leq M<+\infty$ such that $\left(R_{\nu}^{2} / r_{\nu} r_{\nu}^{\prime}\right) \leq M$ (or equivalently $\left.\left(R_{\nu} / r_{\nu}\right)<M^{\prime}<+\infty, r_{\nu} \leq r_{\nu}^{\prime}\right)$

COROLlary 3.5. In Corollary 3.4, the condition $\frac{R^{2} \nu}{r_{\nu} r_{\nu}^{\prime}}<M$ is sufficient but not necessary as is illustrated by the following example:

EXAMPLE. Let $B \subset \mathbb{C}^{2}$ be the unit ball $z_{1} \bar{z}_{1}+z_{2} \bar{z}_{2}<1$ and $z^{(\nu)}=\left(\frac{1}{\nu}, b_{\nu}\right), w^{(\nu)}=$ $\left(\frac{1}{\nu} e^{\frac{\pi}{6}}, b_{\nu}\right), b_{\nu}^{2}=1-\frac{4}{\nu^{2}}, \nu \geq 2$.

If $D_{\nu}=P\left(z^{(\nu)}, w^{(\nu)}\right) \cap B$, then the radius of $D_{\nu}=\frac{2}{\nu}$. Therefore

$$
\rho_{\nu}=\frac{1}{\nu}, \quad \rho_{\nu}^{\prime}=\frac{1}{\nu}, \quad R_{\nu}=\frac{1}{\nu}
$$

Thus, $\frac{R_{\nu}^{2}}{\rho_{\nu} \rho_{\nu}^{\prime}}=1$ for all $\nu$, hence $\left\{C_{B}\left(z^{(\nu)}, w^{(\nu)}\right)\right\}$ is bounded.

In fact, from Corollary 3.1:

$$
C_{B}\left(z^{(\nu)}, w^{(\nu)}\right) \equiv \log \left(\frac{\sqrt{13}+2}{3}\right), \text { for all } \nu
$$


While $r_{\nu}=r_{\nu}^{\prime}<\frac{3}{\nu^{2}}$, thus,

$$
\frac{R_{\nu}^{2}}{r_{\nu} r_{\nu}^{\prime}}>\frac{\nu^{2}}{9} \rightarrow+\infty
$$

as $\nu \rightarrow \infty$. Thus, the condition $\frac{R_{\nu}^{2}}{r_{\nu} r_{\nu}^{\prime}} \leq M<+\infty$ is not necessary for $C_{B}\left(z^{(\nu)}, w^{(\nu)}\right)$ to be bounded.

Proposition 3.1. Let $z, w \in B_{r}, z, w \in N_{z_{o}}, z_{o} \in \partial B_{r},\left|z-z_{o}\right|=r_{1},\left|w-z_{o}\right|=r_{2}$ and $r_{1}<r_{2}<r$. Then

$$
C_{B_{r}}(z, w)=-\frac{1}{2} \log r_{1}+\frac{1}{2} \log r_{2}+\Psi(z, w)
$$

where $|\Psi(z, w)| \leq k<+\infty$

Proof. This is because $N_{z_{o}}$ is a geodesic in $B_{r}$

ThEOREM 3.2. Let $G \subset \mathbb{C}^{n}$ be a strongly pseudoconvex domain and $z_{o} \in \partial G$. If $z, w \in N_{z_{o}}, r_{1}=\left|z-z_{o}\right|, r_{2}=\left|w-z_{o}\right|, r_{1}<r_{2}<r$, where $B_{r}$ is an admissible ball to $G$, then

$$
C_{G}(z, w)=-\frac{1}{2} \log r_{1}+\frac{1}{2} \log r_{2}+\Psi(z, w)
$$

where $|\Psi(z, w)|<k<+\infty$.

Pr o of. From Proposition 3.1:

$$
C_{G}(z, w) \leq C_{B_{r}}(z, w)=-\frac{1}{2} \log r_{1}+\frac{1}{2} \log r_{2}+\Psi(z, w)
$$

$$
|\Psi(z, w)| \leq k_{1}<+\infty
$$

In [4], it is proved that if $A \in G$ is fixed and $\xi \in G$, then

$$
C_{G}(A, \xi)=-\frac{1}{2} \log r^{\prime}+\phi(\xi)
$$

where

$$
|\phi(\xi)| \leq k^{\prime}<+\infty \text { and } r^{\prime}=d(\xi, \partial G)
$$

Thus,

$$
\begin{aligned}
& C_{G}(z, w) \geq C_{G}(A, z)-C_{G}(A, w) \\
= & -\frac{1}{2} \log r_{1}+\frac{1}{2} \log r_{2}+(\phi(z)-\phi(w)) .
\end{aligned}
$$

From (3.1) and (3.2), the result follows.

\section{Domains in $\mathbb{C}^{n}$}

Theorem 4.1. Let $G \subset \mathbb{C}^{n}$ be a smooth domain and $B_{r}, r>0$ be an admissible ball to $G$. Let $\left\{z_{\nu}\right\},\left\{w_{\nu}\right\} \subset G$ such that:

(i) If $L_{\nu}$ is the line joining $z_{\nu}$ to $w_{\nu}$, then $L_{\nu} \subset G$.

(ii) If $\xi \in L_{\nu}$, then $d(\xi, \partial G) \leq r$.

(iii) Let $\lambda_{\nu}=d\left(L_{\nu}, \partial G\right)$, and $\ell_{\nu}=$ length of $L_{\nu}$. If $\left(\ell_{\nu} / \lambda_{\nu}\right) \leq k<+\infty$ for all $\nu$.

Then $\left\{C_{G}\left(z_{\nu}, w_{\nu}\right)\right\}$ will be bounded $\left(\leq 2(k+1) \log \left(\frac{\sqrt{6}+\sqrt{2}}{2}\right)\right)$.

Proof. Let $m=[k]+1,[k]=$ integral part of $k$. 
We divide $L_{\nu}$ into $2 m$ equal parts, each of length $\leq \lambda_{\nu} / 2$ by the points $z_{\nu}=$ $x_{o}, x_{1}, \cdots, x_{2 m}=w_{\nu}$.

Thus,

$$
\begin{gathered}
R_{j}^{\prime}=\left|x_{j+1}-x_{j}\right| \leq \lambda_{\nu} / 2, \quad j=0, \cdots, 2 m-1, \\
r_{j}^{\prime}=d\left(x_{j}, \partial G\right) \geq \lambda_{\nu}, \quad r_{j}^{\prime \prime}=d\left(x_{j+1}, \partial B_{j}\right) \geq \frac{\lambda \nu}{2},
\end{gathered}
$$

(Since $\left(r_{j}^{\prime \prime}+R_{j}^{\prime} \geq r_{j}^{\prime}\right)$, where $B_{j}=B_{r}^{\left(x_{j}\right)}$. Thus

$$
\left(R_{j}^{\prime 2} / r_{j}^{\prime} r_{j}^{\prime \prime}\right) \leq \frac{1}{2}
$$

and thus from Corollary 3.3 we get

$$
C_{G}\left(x_{j}, x_{j+1}\right) \leq C_{B_{j}}\left(x_{j}, x_{j+1}\right) \leq \log \frac{\sqrt{6}+\sqrt{2}}{2},
$$

which proves the theorem.

TheOrem 4.2. Let $G \subset \mathbb{C}^{n}$ be a smooth convex domain $\left\{z_{\nu}\right\}_{\nu=1}^{\infty},\left\{w_{\nu}\right\}_{\nu=1}^{\infty} \subset G$, $r_{\nu}=d\left(z_{\nu}, \partial G\right), r_{\nu}^{\prime}=d\left(w_{\nu}, \partial G\right), \lim _{\nu \rightarrow \infty} z_{\nu}=z_{o}=\lim _{\nu \rightarrow \infty} w_{\nu}, R_{\nu}=\left|z_{\nu}-w_{\nu}\right|$, if $r_{\nu} \leq r_{\nu}^{\prime}, R_{\nu} \leq k r_{\nu}, 0 \leq k<+\infty$ (or equivalently $\frac{R_{\nu}^{2}}{r_{\nu} r_{\nu}^{\prime}} \leq M<+\infty$ ) then,

$$
\left\{C_{G}\left(z_{\nu}, w_{\nu}\right)\right\}
$$

is bounded by $k^{\prime}$.

The condition $r_{\nu} \leq r_{\nu}^{\prime}$ is not a restriction since $C_{G}\left(z_{\nu}, w_{\nu}\right)$ is symmetric in $z_{\nu}$ and $w_{\nu}$.

Proof. Let $L_{\nu}$ and $r_{\nu}$ be as in Theorem 4.1. Since $G$ is convex, $r_{\nu}=d\left(L_{\nu}, \partial G\right)$. Obvious there exists $\nu_{o}$ such that $d(\xi, \partial G)<r$ for $\xi \in L_{\nu}, \nu \geq \nu_{o}$. Thus all the conditions of Theorem 4.1 are satisfied.

COROLlary 4.1. Theorem 4.2 remains valid if $B_{r}$ is an admissible ball to $G$ at $z_{o}$ and $k^{\prime}$ depends on $k$ and $r$.

TheOREM 4.3. Let $G \subset \subset \mathbb{C}^{n}$ be a smooth strongly pseudoconvex, $z_{o} \in \partial G,\left\{z_{\nu}\right\}_{\nu=1}^{\infty}$, $\left\{w_{\nu}\right\}_{\nu=1}^{\infty} \subset G$ be two sequences converging to $z_{o}$ if $r_{\nu}=d\left(z_{\nu}, \partial G\right), r_{\nu}^{\prime}=d\left(w_{\nu}, \partial G\right)$, $R_{\nu}=\left|z_{\nu}-w_{\nu}\right|$. If $\frac{R_{\nu}^{2}}{r_{\nu} r_{\nu}^{\prime}} \leq M<+\infty$ (or equivalently $\left.r_{\nu} \leq r_{\nu}^{\prime},\left(R_{\nu} / r_{\nu}\right) \leq M_{1}<+\infty\right)$ then

$$
\left\{C_{G}\left(z_{\nu}, w_{\nu}\right)\right\}
$$

is bounded by $k_{1}<+\infty$.

Pr o of. There exist [1. page 132 ] neighbourhoods $U$ and $U^{\prime}$ of $z_{o}, U^{\prime} \subset \subset U$ and a biholomorphic map $\phi: U \rightarrow W \subset \mathbb{C}^{n}$ such that $\phi\left(U^{\prime} \cap G\right)=D$ and $D$ is strictly convex.

Let $\phi\left(z_{\nu}\right)=z_{\nu}^{\prime}, \phi\left(w_{\nu}\right)=w_{\nu}^{\prime}, d\left(z_{\nu}^{\prime}, \partial D\right)=\rho_{\nu}, d\left(w_{\nu}^{\prime}, \partial D\right)=\rho_{\nu}^{\prime}, R_{\nu}^{\prime}=\left|z_{\nu}^{\prime}-w_{\nu}^{\prime}\right|$, then from Propositions 2.1, 2.2 there exists $0<k<+\infty$ such that $\frac{R_{\nu}^{\prime 2}}{\rho_{\nu} \rho_{\nu}^{\prime}} \leq k M$. From Theorem 4.2, $\left\{C_{D}\left(z_{\nu}^{\prime}, w_{\nu}^{\prime}\right)\right\}$ will be bounded. Since $C_{G}\left(z_{\nu}, w_{\nu}\right) \leq C_{D}\left(z_{\nu}^{\prime}, w_{\nu}^{\prime}\right)$. The result follows.

We notice that $k_{1}$ depends only on $M_{1}$ and $z_{0}$.

As proved in Section 3, the condition $\frac{R_{\nu}^{2}}{r_{\nu} r_{\nu}^{\prime}} \leq M<+\infty$, is not necessary. 
Theorem 4.4. If in Theorem 4.2, the condition $\left(R_{\nu}^{2} / r_{\nu} r_{\nu}^{\prime}\right)<M$ is replaced by $R_{\nu} \leq \lambda r_{\nu}$ and $\left(R_{\nu} / r_{\nu}^{\prime}\right) \rightarrow \infty$ as $\nu \rightarrow+\infty$, then

$$
C_{G}\left(z_{\nu}, w_{\nu}\right)=-\frac{1}{2} \log r_{\nu}^{\prime}+\frac{1}{2} \log r_{\nu}+\phi\left(z_{\nu}, w_{\mu}\right),
$$

and

$$
\left|\phi\left(z_{\nu}, w_{\nu}\right)\right| \leq k<+\infty
$$

Proof. Let $A \in G$ be fixed. As in Theorem 3.2

$$
\begin{gathered}
C_{G}\left(z_{\nu}, w_{\nu}\right) \geq C_{G}\left(A, w_{\nu}\right)-C_{G}\left(A, z_{\nu}\right) \\
=-\frac{1}{2} \log r_{\nu}^{\prime}+\frac{1}{2} \log r_{\nu}+k_{\nu},
\end{gathered}
$$

where $\left|k_{\nu}\right| \leq k$. Let $w_{\nu}^{\prime}$ be the projection of $w_{\nu}$ on $\partial G$ and $w_{\nu}^{\prime \prime} \in N_{w_{\nu}^{\prime}}$ such that $r_{\nu}^{\prime \prime}=$ $\left|w_{\nu}^{\prime}-w_{\nu}^{\prime \prime}\right|=R_{\nu}+r_{\nu}>R_{\nu}$. Let $R_{\nu}^{\prime}=\left|z_{\nu}-w_{\nu}^{\prime \prime}\right|$, then $R_{\nu}^{\prime} \leq 2 R_{\nu}$. Thus, $\frac{R_{\nu}^{\prime 2}}{r_{\nu} r_{\nu}^{\prime \prime}} \leq 4 \lambda$.

Thus, from Theorem 4.3, $\left\{C_{G}\left(w_{\nu}^{\prime \prime}, z_{\nu}\right)\right\}$ is bounded $<M$. Therefore,

$$
\begin{gathered}
C_{G}\left(z_{\nu}, w_{\nu}\right) \leq C_{G}\left(z_{\nu}, w_{\nu}^{\prime \prime}\right)+C_{G}\left(w_{\nu}, w_{\nu}^{\prime \prime}\right) \\
\leq M-\frac{1}{2} \log r_{\nu}^{\prime}+\frac{1}{2} \log \left(r_{\nu}^{\prime}+R_{\nu}\right)+k_{\nu}^{\prime} \quad(\text { from Theorem 3.2) }
\end{gathered}
$$

$$
\leq M-\frac{1}{2} \log r_{\nu}^{\prime}+\frac{1}{2} \log 2 R_{\nu}+k_{\nu}^{\prime \prime}, \quad \leq M_{\nu}-\frac{1}{2} \log r_{\nu}^{\prime}+\frac{1}{2} \log r_{\nu}
$$

where $M_{\nu}<k_{1}$ for all $\nu$.

From (4.1) and (4.2), we get the result.

Theorem 4.5. In Theorem 4.4 , if Condition $R_{\nu} \leq \lambda r_{\nu}$ is replaced by $\left(R_{\nu} / r_{\nu}\right) \rightarrow+\infty$ then, there exists a constant $k$ such that

$$
C_{G}\left(z_{\nu}, w_{\nu}\right) \leq-\frac{1}{2} \log r_{\nu}-\frac{1}{2} \log r_{\nu}^{\prime}+\log \left(R_{\nu}+r_{\nu}\right)+k
$$

for all $\nu$.

Proof. Let $z_{\nu}^{\prime}$ be the projection of $z_{\nu}$ on $\partial G, z_{\nu}^{\prime \prime} \in N_{z_{\nu}^{\prime}}$ such that $\left|z_{\nu}^{\prime}-z_{\nu}^{\prime \prime}\right|=r_{\nu}^{\prime \prime}=$ $r_{\nu}+R_{\nu}$.

Then $z_{\nu}^{\prime \prime}, w_{\nu}$ satisfy conditions of Theorem 4.3

$$
C_{G}\left(z_{\nu}^{\prime \prime}, w_{\nu}\right)=-\frac{1}{2} \log r_{\nu}^{\prime}+\frac{1}{2} \log \left(r_{\nu}+R_{\nu}\right)+k_{\nu}
$$

Also, from Theorem 3.2

$$
C_{G}\left(z_{\nu}, z_{\nu}^{\prime \prime}\right)=-\frac{1}{2} \log r_{\nu}+\frac{1}{2} \log \left(r_{\nu}+R_{\nu}\right)+k_{\nu}^{\prime}
$$

where $k_{\nu}$ and $k_{\nu}^{\prime}$ are bounded.

By the triangle axiom

$$
\begin{aligned}
C_{G}\left(z_{\nu}, w_{\nu}\right) \leq & -\frac{1}{2} \log r_{\nu}-\frac{1}{2} \log r_{\nu}^{\prime} \\
& +\log \left(r_{\nu}+R_{\nu}\right)+k .
\end{aligned}
$$


Corollary 4.2. If $r_{\nu} \geq r_{\nu}^{\prime}$ then $1 \leq \frac{r_{\nu}+R_{\nu}}{r_{\nu}^{\prime}+R_{\nu}} \leq 2$, (since $r_{\nu}^{\prime}+R_{\nu} \geq r_{\nu}$ ). Thus in Theorem 4.5, there exist $0 \leq k^{\prime}<+\infty$ such that

$$
\begin{aligned}
C_{G}\left(z_{\nu}, w_{\nu}\right) \leq & -\frac{1}{2} \log r_{\nu}-\frac{1}{2} \log r_{\nu}^{\prime}+\frac{1}{2} \log \left(r_{\nu}+R_{\nu}\right) \\
& +\frac{1}{2} \log \left(r_{\nu}^{\prime}+R_{\nu}\right)+k^{\prime}
\end{aligned}
$$

which is the formula obtained before in [6].

Corollary 4.3. From Theorems 3.2, 4.3, 4.4, 4.5 and Corollary 4.2, we see that inequality (4.3) is valid for any two sequences $\left\{z_{\nu}\right\},\left\{w_{\nu}\right\} \subset G$ converging to a point $z_{o} \in \partial G$ where $G$ is a smooth strongly pseudoconvex domain, ( $k^{\prime}$ depends on $\left.z_{o}\right)$.

THEOREM 4.6 (a necessary condition). Let $G \subset \mathbb{C}^{n}$ be a smooth strongly pseudoconvex domain. Let $\left\{z_{\nu}\right\}_{\nu=1}^{\infty},\left\{w_{\nu}\right\}_{\nu=1}^{\infty} \subset G$, for $\left\{C_{G}\left(z_{\nu}, w_{\nu}\right)\right\}$ to be bounded, it is necessary that $0<\ell_{1} \leq \frac{r_{\nu}}{r_{\nu}^{\prime}} \leq \ell_{2}<+\infty$ where $r_{\nu}=d\left(z_{\nu}, \partial G\right), r_{\nu}^{\prime}=d\left(w_{\nu}, \partial G\right)$.

Proof. Let $A \in G$ be a fixed point. Then [4]

$$
C_{G}\left(A, z_{\nu}\right)=-\frac{1}{2} \log r_{\nu}+k\left(z_{\nu}\right)
$$

where $|k(z)| \leq k_{1}<+\infty$, for all $z \in G$.

Since,

$$
C_{G}\left(z_{\nu}, w_{\nu}\right) \geq\left|C_{G}\left(A, z_{\nu}\right)-C_{G}\left(A, w_{\nu}\right)\right|
$$

we get the result.

ExAMPLE. We give an example to show that in any smooth strongly pseudoconvex domain $G \subset \mathbb{C}^{n}$, there exist sequences $\left\{z_{\nu}\right\},\left\{w_{\nu}\right\} \subset G$ converging to a point $z_{o} \in \partial G$, $\lim _{\nu \rightarrow \infty} \frac{R_{\nu}}{r_{\nu}}=\lim _{\nu \rightarrow \infty} \frac{R_{\nu}}{r_{\nu}^{\prime}}=+\infty$ and in spite of this $\left\{C_{G}\left(z_{\nu}, w_{\nu}\right)\right\}$ is bounded; i.e., the condition $\frac{R_{\nu}^{2}}{r_{\nu} r_{\nu}^{\prime}}<k<+\infty$ is not necessary for the boundedness of $\left\{C_{G}\left(z_{\nu}, w_{\nu}\right)\right\}$.

We use the idea of the example given in Section 3. Let $B=B_{r} \subset \mathbb{C}^{n}$ be an admissible ball to $G, \zeta_{o} \in \partial G$ and $\left\{\zeta_{\nu}\right\}_{\nu=1}^{\infty} \subset \partial G$ converging to $\zeta_{o}$.

Let $z_{\nu}, w_{\nu} \in B, z_{\nu}=\left\{\frac{r}{\nu}, b_{\nu}, 0, \cdots, 0\right\}, w_{\nu}=\left\{\frac{r}{\nu} e^{i \frac{\pi}{6}}, b_{\nu}, 0, \cdots, 0\right\}, \nu=2,3, \cdots, b_{\nu}^{2}=$ $r^{2}-\frac{4 r^{2}}{\nu^{2}}$.

As in section $3, C_{B}\left(z_{\nu}, w_{\nu}\right)=\log \left(\frac{\sqrt{13}+2}{3}\right)$ for all $\nu$.

Let $z_{\nu}^{\prime}$ be the projection of $z_{\nu}$ onto $\partial B$. Let $B_{z_{\nu}^{\prime}}$ be the ball $B$ placed tangential to $\partial G$ at $\zeta_{\nu}$ with the point $z_{\nu}^{\prime}$ coincident with $\zeta_{\nu}$ such that the diameter of $B$ through $z_{\nu}^{\prime}, z_{\nu}$ lie on $N_{\zeta_{\nu}}$. Let $z_{\nu}, w_{\nu}$ coincide with $z_{\nu}^{\prime \prime}, w_{\nu}^{\prime} \in G$. Obvious $z_{\nu}^{\prime \prime} \rightarrow \zeta_{o}$ and $w_{\nu}^{\prime} \rightarrow \zeta_{o}$. Now

$$
\left|z_{\nu}-z_{\nu}^{\prime}\right|=\left|\zeta_{\nu}-z_{\nu}^{\prime \prime}\right|=d\left(z_{\nu}, \partial B\right)=d\left(z_{\nu}^{\prime \prime}, \partial G\right)=r_{\nu}
$$

and

$$
R_{\nu}=\left|z_{\nu}-w_{\nu}\right|=\left|z_{\nu}^{\prime \prime}-w_{\nu}\right| .
$$

As proved in the example in section $3, \frac{R_{\nu}}{r_{\nu}} \rightarrow+\infty$.

It is obvious that

$$
C_{G}\left(z_{\nu}^{\prime \prime}, w_{\nu}^{\prime}\right) \leq C_{B}\left(z_{\nu}, w_{\nu}\right)=\log \left(\frac{\sqrt{13}+2}{3}\right)
$$


Let $r_{\nu}^{\prime}=d\left(w_{\nu}^{\prime}, \partial G\right)$. Since $C_{G}\left(z_{\nu}^{\prime \prime}, w_{\nu}^{\prime}\right)$ is bounded, then from Theorem 4.5, we get

$$
0<\ell_{1} \leq \frac{r_{\nu}}{r_{\nu}^{\prime}} \leq \ell_{2}<+\infty
$$

Since $\left(R_{\nu} / r_{\nu}\right) \rightarrow \infty$, then

$$
\left(R_{\nu} / r_{\nu}^{\prime}\right) \rightarrow+\infty
$$

5. Continuous (topological) extensions of biholomorphic maps of strongly pseudoconvex domains

\section{DEFINITION 5.1.}

Let $G \subset \subset \mathbb{C}^{n}$ be a smooth domain:

(i) Let $z, w \in G, r_{1}=d(z, \partial G), r_{2}=d(w, \partial G)$. We define

$$
\Psi_{G}(z, w)=-\frac{1}{2} \log r_{1}-\frac{1}{2} \log r_{2},
$$

and

$$
T_{G}(z, w)=\Psi_{G}(z, w)-C_{G}(z, w)
$$

(ii) Let $S=\left\{z_{\nu}\right\}_{\nu=1}^{\infty} \subset G$. $S$ is called a boundary sequence if $S$ has no limiting point in $G$. Furthermore, if $\lim _{\nu \rightarrow \infty} z_{\nu}=z_{o} \in \partial G, S$ is called a simple boundary sequence.

Now, let $G$ be strongly pseudoconvex, $\left\{z_{\nu}\right\}_{\nu=1}^{\infty},\left\{w_{\nu}\right\}_{\nu=1}^{\infty} \subset G$ and $\lim _{\nu \rightarrow \infty} z_{\nu}=\lim _{\nu \rightarrow \infty} w_{\nu}$ $=z_{o} \in \partial G$, then from Corollary 4.3, we get

$$
\lim _{\nu \rightarrow \infty} T_{G}\left(z_{\nu}, w_{\nu}\right)=+\infty
$$

In [5], it is proved that if $z_{\nu} \rightarrow z_{o} \in \partial G$ and $w_{\nu} \rightarrow w_{o} \in \partial G, z_{o} \neq w_{o}$, there exists $\nu_{o}>0$, such that

$$
C_{G}\left(z_{\nu}, w_{\nu}\right)=-\frac{1}{2} \log r_{\nu}-\frac{1}{2} \log r_{\nu}^{\prime}+\theta\left(z_{\nu}, w_{\nu}\right)
$$

where $r_{\nu}=d\left(z_{\nu}, \partial G\right), r_{\nu}^{\prime}=d\left(w_{\nu}, \partial G\right)$ and $\left|\theta\left(z_{\nu}, w_{\nu}\right)\right| \leq k<+\infty$ for all $\nu \geq \nu_{o}$. Thus,

$$
\left|T_{G}\left(z_{\nu}, w_{\nu}\right)\right| \leq k^{\prime} \text { for all } \nu
$$

From (5.1) and (5.2), we see that if:

(i) $\left\{z_{\nu}\right\} \subset G$ is simple boundary sequence $\rightarrow z_{o} \in \partial G$,

(ii) $\left\{w_{\nu}\right\} \subset G$ is a boundary sequence then,

$$
\lim _{\nu \rightarrow \infty} T_{G}\left(z_{\nu}, w_{\nu}\right)=+\infty
$$

if and only if $\left\{w_{\nu}\right\} \subset G$ is a simple boundary sequence $\rightarrow z_{o}$.

Now, let $\phi$ be a biholomorphic map of $G$ onto another smooth strongly pseudoconvex domain $G_{1}, \phi(z)=x \in G_{1}, r=d(z, \partial G), \rho=d\left(x, \partial G_{1}\right), A \in G$ be a fixed point and $\phi(A)=A^{\prime}$. In [4], it is proved that

$$
\begin{gathered}
C_{G}(A, z)=-\frac{1}{2} \log r+k(z), \quad|k(z)|<k_{1}, \quad \text { for all } z \in G \\
C_{G_{1}}\left(A^{\prime}, x\right)=-\frac{1}{2} \log \rho+k^{\prime}(x), \quad\left|k^{\prime}(x)\right|<k_{1}^{\prime}, \text { for all } x \in G_{1}
\end{gathered}
$$

Since

$$
C_{G}(A, z)=C_{G_{1}}\left(A^{\prime}, x\right)
$$


there exist $0<\ell_{1}<\ell_{2}<+\infty$ such that $\ell_{1} \leq(r / \rho) \leq \ell_{2}$ for all $z \in G, x \in G_{1}$.

Thus, if $w \in G, \phi(w)=y$

$$
\left|\Psi_{G_{1}}(x, y)-\Psi_{G}(z, w)\right|<k_{3} \quad \text { for all } z, w \in G,
$$

Thus,

$$
\left|\left[\Psi_{G_{1}}(x, y)-C_{G_{1}}(x, y)\right]-\left[\Psi_{G}(z, w)-C_{G}(z, w)\right]\right|<k_{3}
$$

i.e.,

$$
\left|T_{G_{1}}(x, y)-T_{G}(z, w)\right|<k_{3}
$$

Now, let $\left\{z_{\nu}\right\} \subset G$ be any simple boundary sequence $\rightarrow z_{o} \in \partial G$, such that $\left\{x_{\nu}=\right.$ $\left.\phi\left(z_{\nu}\right)\right\}_{\nu=1}^{\infty} \subset G_{1}$ be also a simple boundary sequence $\rightarrow x_{o} \in \partial G_{1}$

Furthermore, let $\left\{w_{\nu}\right\}_{\nu=1}^{\infty} \subset G$ be any simple boundary sequence $\rightarrow z_{o} \in \partial G$. Thus, from (5.1)

$$
\lim _{\nu \rightarrow \infty} T_{G}\left(z_{\nu}, w_{\nu}\right)=+\infty
$$

From (5.3) if $y_{\nu}=\phi\left(w_{\nu}\right)$, then

$$
\lim _{\nu \rightarrow \infty} T_{G_{1}}\left(x_{\nu}, y_{\nu}\right)=+\infty
$$

Since $\left\{x_{\nu}\right\} \subset G_{1}$ is a simple boundary sequence $\rightarrow x_{o}$, then $\left\{y_{\nu}\right\}$ will be a simple boundary sequence $\rightarrow x_{o}$.

Doing the same thing with $\phi^{-1}$, we see that if $\left\{y_{\nu}\right\}_{\nu=1}^{\infty} \subset G_{1}$ is any boundary sequence converging to $x_{o}$, then $\left\{w_{\nu}=\phi^{-1}\left(y_{\nu}\right)\right\}_{\nu=1}^{\infty}$ will be also a simple boundary sequence converging to $z_{o}$. Thus if we define $\phi\left(z_{o}\right)=w_{o}$, we get the following theorem.

THEOREM 5.1. Any biholomorphic map of a strongly pseudoconvex domain $G \subset \mathbb{C}^{n}$ with a $C^{2}$ boundary onto a strongly pseudoconvex domain $G_{1} \subset \mathbb{C}^{n}$ with a $C^{2}$ boundary, has a topological extension to be boundary.

\section{References}

[1] M. Abate, Iteration theory of holomorphic maps on taut manifolds, Italy: Mediterranean Press, 1989.

[2] C. Carathéodory, Über eine spezielle Metrik, die in der Theorie der analytischen Funktionen auftritt, Atti Pontif. Accad. Sci. Nuovi Lincei 80 (1927), 135-141.

[3] A. A. Fadlalla, On the group of automorphism of the Euclidean hypersphere, Mathematika 15 (1968), 193-198.

[4] A. A. Fadlalla, On the boundary behaviour of the Carathéodory and Kobayashi distances in strongly pseudoconvex domains in $\mathbb{C}^{n}$, Proc. Int. Workshop in Wuppertal (1990), Aspects of Mathematics (1990), 111-114.

[5] A. A. Fadlalla, The Carathéodory distance in strongly pseudoconvex domains, Math. Ann. 298 (1994), 141-144.

[6] F. Forestneric, J. P. Rosay, Localization of the Kobayashi metric and the boundary continuity of proper holomorphic mappings, Math. Ann. 279 (1987), 239-252.

[7] E. Vesentini, Complex geodesic, Compositio Math. 44 (1981), 375-394.

[8] N. Vormoor, Topologische Fortsetzung biholomorpher Funktionen auf den Rand bei beschränkten streng-pseudokonvexen Gebieten in $\mathbb{C}^{n}$, Math. Ann. 204 (1973), 239-261. 